Review began 12/13/2021 Review ended 12/22/202 Published 12/25/2021

(๑) Copyright 2021

Al Zabali et al. This is an open access article distributed under the terms of the Creative Commons Attribution License $\mathrm{CC}$ BY 4.0., which permits unrestricted use distribution, and reproduction in any medium, provided the original author an source are credited.

\section{Brucella Peritonitis in a Patient on Peritoneal Dialysis: A Case Report and Review of Literature}

Saeed M. Al Zabali ${ }^{1}$, Aljawharah K. Rubaihan ${ }^{2}$, Madawi F. Alnetaifat ${ }^{3}$, Salem Alshahrani ${ }^{4}$, Moza Alhammadi 5, 6

1. Pediatric Nephrology, King Fahad Medical City, Riyadh, SAU 2. College of Medicine, Al-Maarefa University, Riyadh, SAU 3. College of Medicine, King Saud Bin Abdulaziz University for Health Sciences, Riyadh, SAU 4. College of Medicine, King Khalid University, Asir-Abha, SAU 5. Pediatric Infectious Diseases, Dubai Hospital, Dubai Health Authority, Dubai, ARE 6. Pediatric Infectious Diseases, King Fahad Medical City, Riyadh, SAU

Corresponding author: Saeed M. Al Zabali, alzablis@ymail.com

\begin{abstract}
Peritoneal dialysis (PD)-associated peritonitis is the most common cause of morbidity, mortality, and treatment failure in patients on PD. Brucellosis is a worldwide zoonotic infectious disease caused by gramnegative bacteria of the genus Brucella. It is a major public issue in some regions. According to the World Health Organization report in 2011, the Kingdom of Saudi Arabia is considered endemic for brucellosis. Brucella peritonitis is one of the rarest presentations of Brucella. We report a case of a 14-year-old girl known to have end-stage renal disease, secondary to the autosomal recessive polycystic kidney. She had congenital hepatic fibrosis and pancytopenia. She had been undergoing automated PD for the past seven years and presented with abdominal pain, seizure, and poor feeding. There was no history of ingestion of unpasteurized milk or contact with raw infected animal products. The color of PD fluid was turbid with leukocytosis, predominantly neutrophils. The peritoneal fluid culture was positive for methicillin-resistant Staphylococcus aureus. The patient was started on intraperitoneal vancomycin, which showed slow improvement. The second culture of the peritoneal fluid showed Brucella species after a few days. Blood culture and serum serology titer for Brucella showed negative results. An anti-Brucella regimen, including rifampin and doxycycline, was initiated. She was treated with this regimen for six weeks. After the initiation of the anti-Brucella regimen, she showed marked improvement. To the best of our knowledge, only a small number of cases of Brucella peritonitis in PD patients have been reported. Despite the rarity of Brucella as a peritonitis-causing organism, it should be considered as a relevant pathogen in peritonitis cases, especially in endemic regions. PD-associated Brucella peritonitis is rare, and PD catheter saving may be considered if there is a response to anti-Brucella treatment.
\end{abstract}

Categories: Pediatrics, Nephrology

Keywords: end-stage renal disease, catheter removal, peritoneal dialysis, peritonitis, brucella

\section{Introduction}

Peritoneal dialysis (PD) is the primary modality of renal replacement therapy in children [1]. The number of patients with end-stage renal disease (ESRD) receiving PD therapy has been increasing because of the improvement in PD techniques and patient survival [2]. PD-associated peritonitis is a major cause of hospitalization in pediatric patients [3]. Peritonitis is the most common complication of PD; it is generally caused by coagulase-negative Staphylococcus and Staphylococcus aureus [4]. Brucellosis, also known as "undulant fever" or "Mediterranean fever," is a zoonotic infectious disease transmitted primarily by direct or indirect contact with infected animals or their products. Infection generally occurs in endemic areas [5]. Brucellosis is endemic in Saudi Arabia, with an incidence of 12.44 cases per 100,000 population as reported in 2019 [4]. The bacterium Brucella first infects animals and is then transmitted to humans through contact with an infected animal [6]. Brucellosis generally presents as an acute or subacute infection. Hepatosplenomegaly, fever, and peripheral arthritis are the most frequent clinical findings [5]. Peritonitis caused by brucellosis is considered to be rare. Most reported cases were in Saudi Arabia and Turkey, with only one case reported in China. Herein, we present a case of Brucella-related PD in a patient who was treated with doxycycline and rifampin for six weeks without the removal of the PD catheter.

\section{Case Presentation}

The patient is a 14-year-old Saudi girl with congenital hepatic fibrosis with pancytopenia and ESRD, secondary to autosomal recessive polycystic kidney disease, on automated PD for seven years. She had a medical history of seizure disorders and developmental delay and was on antiepileptic drugs. She was presented to the emergency department with complaints of abdominal pain for one day and a change in the peritoneal dialysate color, associated with abnormal movements described as generalized tonic-clonic convulsions. She had no fever or vomiting, but there was a history of acquiring bacterial peritonitis infections several times. She received a blood transfusion a few months before the presentation and was not known to have any allergies. Her vital signs at admission were as follows: temperature: $37.2^{\circ} \mathrm{C}$, heart rate: 106/min, respiratory rate: $24 / \mathrm{min}$, and blood pressure: $97 / 32 \mathrm{mmHg}$. On examination, she was conscious and alert but irritable and appeared pale. Her abdomen was distended with tenderness throughout. Other systemic examinations were unremarkable. Upon presentation, the patient's white blood cell (WBC) count was low at $3.0910 \mathrm{e} 9 / \mathrm{L}$, along with a high neutrophil proportion of $79.6 \%$, low lymphocyte proportion of $11.0 \%$, a low hemoglobin level of $8.7 \mathrm{~g} / \mathrm{dl}$, low platelet count of $61.010 \mathrm{e} / \mathrm{L}$ (it is the baseline for this case), markedly elevated plasma creatinine level of $675 \mathrm{mmol} / \mathrm{L}$, high urea level of $21 \mathrm{mmol} / \mathrm{L}$, bicarbonate (CO2) level of $18 \mathrm{mmol} / \mathrm{L}$, elevated C-reactive protein level of $157 \mathrm{ml} / \mathrm{L}$, high alkaline phosphatase level of 909 U/I, high alanine aminotransferase level of $98 \mathrm{U} / \mathrm{I}$, and a high bilirubin level of $16.4 \mathrm{umol} / \mathrm{L}$. 


\section{Cureus}

A sample of peritoneal fluid was collected for analysis, which exhibited a turbid yellowish appearance, a WBC count of $59,658 / \mathrm{mm}^{3}$, and a high neutrophil proportion of $94 \%$. The peritoneal fluid culture was positive for methicillin-resistant $S$. aureus. The patient was started on intraperitoneal vancomycin and ceftazidime, but the abdominal pain did not decrease with the treatment, and she had one spike of fever of $38.1^{\circ} \mathrm{C}$. Fungal culture was negative and the second culture of peritoneal fluid revealed Brucella species after four days of growth (Table 1).

\begin{tabular}{|l|l|}
\hline Culture & Organism \\
\hline Brucella spp. & \\
Antibiotic sensitivity & Interpretation result \\
Trimethoprim/sulfamethoxazole & Sensitive \\
Gentamicin & Sensitive \\
Streptomycin & Sensitive \\
\hline Tetracycline & Sensitive \\
\hline Doxycycline & Sensitive \\
\hline
\end{tabular}

TABLE 1: Results of peritoneal fluid culture.

Blood culture and serum serology (enzyme-linked immunosorbent assay [ELISA] assay test) for Brucella showed negative results. When we further examined the patient's history, the mother denied any history of ingestion of unpasteurized milk or contact with raw infected animal products. The infectious diseases team was consulted and they started a treatment regimen for brucellosis with oral rifampicin $10 \mathrm{mg} / \mathrm{kg}$ once daily and doxycycline $2 \mathrm{mg} / \mathrm{kg} /$ dose every 12 hours for six weeks. Blood culture showed negative results for Brucella, and a repeat peritoneal culture showed negative findings on the fourth day after treatment initiation. Peritoneal fluid analysis showed a decreased WBC count of $40,000 / \mathrm{mm}^{3}$ and clear peritoneal fluid. Although removal of the PD catheter was indicated, the patient's mother refused as the patient showed clinical improvement. She was discharged in a good condition without the removal of the PD catheter, and PD was resumed as usual without stopping it.

\section{Discussion}

Brucella species are gram-negative unencapsulated, nonmotile, nonspore-forming, facultative intracellular bacilli [7]. They are transmitted to humans from infected animals through ingestion, inhalation, conjunctiva, or skin abrasions. This bacterium affects multiple organs in the body through hematogenous spread [8]. The accurate diagnosis of brucellosis is difficult because of nonspecific clinical characteristics, slow growth in cultures, and the complexity of serological diagnosis [9]. Bone and joint involvement is the most frequent complication of brucellosis. According to the International Society for Peritoneal Dialysis, peritonitis must always be diagnosed when at least two of the following findings are present: (a) clinical features consistent with peritonitis, (b) dialysis effluent WBC count of $>100 / \mu \mathrm{L}$ or $>0.1 \times 109 / \mathrm{L}$ with $>50 \%$ polymorphonuclear cells, and (c) positive dialysis fluid culture [10].

Patients who are on PD are at a higher risk of developing peritonitis than the general population because of the impaired peritoneal defenses in patients on PD [11]. Brucella shunt infection complicated by peritonitis has been reported [12]. PD-associated Brucella peritonitis is extremely rare [8]. To our knowledge, only nine cases have been reported in the literature to date. The majority of the reported cases were in Turkey and Saudi Arabia, with one case reported in China. Table 2 summarizes the general characteristics and clinical findings of the reported cases of Brucella PD-related peritonitis. Almost all the reported cases were adult patients (Table 2). Our patient is the only pediatric case.

\begin{tabular}{|c|c|c|c|c|c|c|c|c|c|c|}
\hline Study & $\begin{array}{l}\text { Taskapan et } \\
\text { al. (2002) [7] }\end{array}$ & $\begin{array}{l}\text { Ozisik et al. } \\
\text { (2006) [11] }\end{array}$ & $\begin{array}{l}\text { Alothman et } \\
\text { al. (2008) } \\
{[13]}\end{array}$ & $\begin{array}{l}\text { Unal et al. } \\
\text { (2009) case } 1 \\
{[8]}\end{array}$ & $\begin{array}{l}\text { Unal et al. } \\
\text { (2009) case } 2 \\
{[8]}\end{array}$ & $\begin{array}{l}\text { Solak et al. } \\
\text { (2012) [14] }\end{array}$ & $\begin{array}{l}\text { Koz et al. } \\
\text { (2014) [15] }\end{array}$ & $\begin{array}{l}\text { Niu et al. } \\
\text { (2018) [16] }\end{array}$ & $\begin{array}{l}\text { Bukhari et al. } \\
\text { (2018) [17] }\end{array}$ & $\begin{array}{l}\text { Pres } \\
\text { repo }\end{array}$ \\
\hline Age, gender & $\begin{array}{l}\text { 47-year-old } \\
\text { male }\end{array}$ & $\begin{array}{l}\text { 39-year-old } \\
\text { woman }\end{array}$ & $\begin{array}{l}\text { 67-year-old } \\
\text { male }\end{array}$ & $\begin{array}{l}\text { 38-year-old } \\
\text { male }\end{array}$ & $\begin{array}{l}\text { 52-year-old } \\
\text { male }\end{array}$ & $\begin{array}{l}\text { 48-year-old } \\
\text { man }\end{array}$ & $\begin{array}{l}\text { 49-year-old } \\
\text { man }\end{array}$ & $\begin{array}{l}\text { 54-year-old } \\
\text { woman }\end{array}$ & $\begin{array}{l}\text { 45-year-old } \\
\text { man }\end{array}$ & $\begin{array}{l}14-y \epsilon \\
\text { wom: }\end{array}$ \\
\hline \multirow[t]{2}{*}{ Country } & Turkey & Turkey & Saudi Arabia & Turkey & Turkey & Turkey & Turkey & China & Saudi Arabia & Saud \\
\hline & $\begin{array}{l}\text { 15-day history } \\
\text { of fatigue, } \\
\text { fever, } \\
\text { sweating, back }\end{array}$ & $\begin{array}{l}\text { Nausea and } \\
\text { severe } \\
\text { abdominal }\end{array}$ & $\begin{array}{l}\text { Change of } \\
\text { peritoneal } \\
\text { fluid color, } \\
\text { associated } \\
\text { with }\end{array}$ & $\begin{array}{l}\text { Two-day } \\
\text { history of } \\
\text { nausea, } \\
\text { vomiting, }\end{array}$ & $\begin{array}{l}\text { nistory of } \\
\text { nausea, } \\
\text { vomiting, } \\
\text { fever, joint }\end{array}$ & $\begin{array}{l}\text { Abdominal } \\
\text { bloating and } \\
\text { constipation } \\
\text { of two weeks, }\end{array}$ & $\begin{array}{l}\text { Abdominal } \\
\text { pain and } \\
\text { cloudy } \\
\text { dialysate }\end{array}$ & $\begin{array}{l}\text { Abdominal } \\
\text { pain, } \\
\text { nausea, } \\
\text { vomiting, } \\
\text { diarrhea, } \\
\text { fatigue, } \\
\text { anorexia, } \\
\text { bilateral }\end{array}$ & $\begin{array}{l}\text { Two to three } \\
\text { days history } \\
\text { of fever, on- } \\
\text { off vague } \\
\text { generalized }\end{array}$ & $\begin{array}{l}\text { Abdo } \\
\text { pain } \\
\text { day a } \\
\text { histol } \\
\text { chan }\end{array}$ \\
\hline
\end{tabular}




\begin{tabular}{|c|c|c|c|c|c|c|c|c|c|c|c|}
\hline \multicolumn{2}{|l|}{ History } & $\begin{array}{l}\text { pain, and two- } \\
\text { day history of } \\
\text { cloudy } \\
\text { dialysate }\end{array}$ & $\begin{array}{l}\text { pain. History } \\
\text { of four } \\
\text { peritonitis } \\
\text { episodes }\end{array}$ & $\begin{array}{l}\text { abdominal } \\
\text { pain and } \\
\text { increased } \\
\text { lower limb } \\
\text { edema of } \\
\text { one week }\end{array}$ & $\begin{array}{l}\text { abdominal } \\
\text { pain, and } \\
\text { cloudy } \\
\text { dialysate }\end{array}$ & $\begin{array}{l}\text { pain, severe } \\
\text { abdominal } \\
\text { pain, and } \\
\text { cloudy } \\
\text { dialysate }\end{array}$ & $\begin{array}{l}\text { no fever or } \\
\text { hypotension, } \\
\text { mild } \\
\text { abdominal } \\
\text { tenderness }\end{array}$ & $\begin{array}{l}\text { effluent. He } \\
\text { had myalgia } \\
\text { and malaise } \\
\text { for } 10 \text { days }\end{array}$ & $\begin{array}{l}\text { knee pain, } \\
\text { cloudy PD } \\
\text { effluent, and } \\
\text { ultrafiltration } \\
\text { decrease, } \\
\text { only one } \\
\text { previous } \\
\text { peritonitis } \\
\text { episode }\end{array}$ & $\begin{array}{l}\text { dull } \\
\text { abdominal } \\
\text { pain, } \\
\text { vomiting, and } \\
\text { diarrhea }\end{array}$ & $\begin{array}{l}\text { peritc } \\
\text { dialy: } \\
\text { color } \\
\text { histol } \\
\text { fever }\end{array}$ \\
\hline \multicolumn{2}{|c|}{ Examination } & $\begin{array}{l}\text { Temperature } \\
\text { of } 38.2^{\circ} \mathrm{C} \text {, } \\
\text { heart rate of } \\
106 \text { beats } / \mathrm{min} \text {, } \\
\text { and blood } \\
\text { pressure of } \\
150 / 100 \\
\mathrm{mmHg} \text {, with } \\
\text { prominent } \\
\text { abdominal } \\
\text { tenderness }\end{array}$ & $\begin{array}{l}\text { Temperature } \\
\text { of } 37.3^{\circ} \mathrm{C} \text {, } \\
\text { blood } \\
\text { pressure of } \\
160 / 90 \\
\mathrm{mmHg} \text {, and } \\
\text { pulse rate of } \\
105 \\
\text { beats/min. } \\
\text { The patient } \\
\text { had } \\
\text { abdominal } \\
\text { pain during } \\
\text { palpation, and } \\
\text { the peritoneal } \\
\text { catheter was } \\
\text { normal }\end{array}$ & $\begin{array}{l}\text { Abdominal } \\
\text { examination } \\
\text { revealed } \\
\text { diffuse mild } \\
\text { tenderness } \\
\text { with clean } \\
\text { CAPD } \\
\text { catheter exit- } \\
\text { site. There } \\
\text { was bilateral } \\
\text { lower limb } \\
\text { edema }\end{array}$ & $\begin{array}{l}\text { Temperature } \\
\text { of } 37.3^{\circ} \mathrm{C} \text {, } \\
\text { heart rate of } \\
88 \text { beats } / \mathrm{min} \text {, } \\
\text { blood } \\
\text { pressure of } \\
135 / 85 \\
\mathrm{mmHg} \text {, and } \\
\text { diffuse } \\
\text { abdominal } \\
\text { mild } \\
\text { tenderness }\end{array}$ & $\begin{array}{l}\text { Temperature } \\
\text { of } 37.2^{\circ} \mathrm{C} \text {, } \\
\text { heart rate of } \\
94 \text { beats/min, } \\
\text { blood } \\
\text { pressure of } \\
150 / 100 \\
\mathrm{mmHg} \text {. The } \\
\text { patient was } \\
\text { pale with } \\
\text { diffuse } \\
\text { abdominal } \\
\text { mild } \\
\text { tenderness } \\
\text { without } \\
\text { rebound } \\
\text { tenderness or } \\
\text { guarding. He } \\
\text { also had } \\
\text { bilateral } \\
\text { orbital edema }\end{array}$ & $\begin{array}{l}\text { No fever or } \\
\text { hypotension, } \\
\text { mild } \\
\text { abdominal } \\
\text { tenderness. } \\
\text { Other aspects } \\
\text { of the physical } \\
\text { examination } \\
\text { were } \\
\text { unremarkable. } \\
\text { Peritoneal } \\
\text { effluent was } \\
\text { not cloudy }\end{array}$ & $\begin{array}{l}\text { No fever. } \\
\text { Physical } \\
\text { examination } \\
\text { revealed } \\
\text { abdominal } \\
\text { tenderness, } \\
\text { negative } \\
\text { rebound } \\
\text { tenderness }\end{array}$ & $\begin{array}{l}\text { Temperature } \\
\text { of } 36.2^{\circ} \mathrm{C} \text {, } \\
\text { blood } \\
\text { pressure of } \\
150 / 80 \\
\mathrm{mmHg} \text {. The } \\
\text { patient had } \\
\text { umbilical } \\
\text { tenderness } \\
\text { during } \\
\text { palpation } \\
\text { and slight } \\
\text { bilateral } \\
\text { lower limb } \\
\text { edema }\end{array}$ & $\begin{array}{l}\text { Temperature } \\
\text { was } 38^{\circ} \mathrm{C} \text {, } \\
\text { blood } \\
\text { pressure was } \\
120 / 60 \\
\mathrm{mmHg} \text {, and } \\
\text { tender } \\
\text { abdomen with } \\
\text { turbid } \mathrm{PD} \text { fluid }\end{array}$ & $\begin{array}{l}\text { Vitall } \\
\text { was } \\
\text { hypo } \\
\text { afebr } \\
\text { exam } \\
\text { she v } \\
\text { irritat } \\
\text { abdo } \\
\text { was } \\
\text { distel } \\
\text { with } \\
\text { tende } \\
\text { all ov } \\
\text { syste } \\
\text { exam } \\
\text { were } \\
\text { unrer }\end{array}$ \\
\hline \multicolumn{2}{|c|}{ Risk factors } & $\begin{array}{l}\text { History of } \\
\text { unpasteurized, } \\
\text { unsalted } \\
\text { cheese } \\
\text { ingestion }\end{array}$ & $\begin{array}{l}\text { Direct contact } \\
\text { with animal }\end{array}$ & $\begin{array}{l}\text { History of } \\
\text { raw milk } \\
\text { ingestion }\end{array}$ & $\begin{array}{l}\text { History of } \\
\text { unpasteurized } \\
\text { cheese } \\
\text { ingestion }\end{array}$ & $\begin{array}{l}\text { History of } \\
\text { unpasteurized } \\
\text { milk and } \\
\text { cheese } \\
\text { ingestion }\end{array}$ & $\begin{array}{l}\text { Contact with } \\
\text { sheep and } \\
\text { cattle }\end{array}$ & Not reported & $\begin{array}{l}\text { Possible } \\
\text { ingestion of } \\
\text { undercooked } \\
\text { beef, single } \\
\text { PD } \\
\text { exchange } \\
\text { while in } \\
\text { restaurant }\end{array}$ & $\begin{array}{l}\text { History of } \\
\text { unpasteurized } \\
\text { cheese } \\
\text { ingestion }\end{array}$ & $\begin{array}{l}\text { No hi } \\
\text { direc } \\
\text { with : } \\
\text { or ral } \\
\text { inges }\end{array}$ \\
\hline \multicolumn{2}{|c|}{ Time to peritonitis } & 12 months & 5 years & 4 months & 2 months & 6 months & 3 years & Not reported & 11 years & 3 years & 7 yez \\
\hline \multirow[t]{2}{*}{$\begin{array}{l}\text { Blood } \\
\text { test }\end{array}$} & Culture & $\begin{array}{l}\text { Brucella } \\
\text { melitensis on } \\
\text { the sixth day }\end{array}$ & No growth & No growth & $\begin{array}{l}\text { Brucella } \\
\text { melitensis on } \\
\text { the fifth day }\end{array}$ & $\begin{array}{l}\text { Brucella } \\
\text { organism on } \\
\text { the sixth day }\end{array}$ & No growth & Not reported & No growth & $\begin{array}{l}\text { Isolated } \\
\text { heavy growth } \\
\text { of Brucella } \\
\text { melitensis on } \\
\text { the fourth day }\end{array}$ & No gi \\
\hline & WBC & $6200 / \mathrm{mm} 3$ & 14.5 & $\begin{array}{l}8.1 \times 109 \\
\text { cells/L }\end{array}$ & $4080 / \mathrm{mm} 3$ & $8100 / \mathrm{mm} 3$ & $\begin{array}{l}7.16 \times 109 \\
\text { cells/L }\end{array}$ & $7100 / \mathrm{mm} 3$ & $\begin{array}{l}17.16 \times 109 \\
\text { cells/L }\end{array}$ & $\begin{array}{l}15.9 \times 109 \\
\text { cells } / L\end{array}$ & 3.09 \\
\hline \multirow{3}{*}{$\begin{array}{l}\text { PD fluid } \\
\text { analysis }\end{array}$} & Culture & $\begin{array}{l}\text { Brucella } \\
\text { melitensis on } \\
\text { the sixth day }\end{array}$ & $\begin{array}{l}\text { Brucella } \\
\text { melitensis }\end{array}$ & $\begin{array}{l}\text { Grew } \\
\text { Brucella spp. } \\
\text { on day six }\end{array}$ & $\begin{array}{l}\text { Brucella } \\
\text { melitensis on } \\
\text { the fifth day }\end{array}$ & $\begin{array}{l}\text { Brucella } \\
\text { organism on } \\
\text { the sixth day }\end{array}$ & Brucella spp. & Brucella spp. & Brucella spp. & $\begin{array}{l}\text { Isolated } \\
\text { heavy growth } \\
\text { of Brucella } \\
\text { melitensis on } \\
\text { the fourth day }\end{array}$ & Bruce \\
\hline & $\begin{array}{l}\text { WBC } \\
\text { differential }\end{array}$ & NA & $\begin{array}{l}\text { Neutrophils } \\
\text { predominance }\end{array}$ & $\begin{array}{l}8 \% \\
\text { lymphocytes } \\
\text { and } 85 \% \\
\text { neutrophils }\end{array}$ & $\begin{array}{l}\text { Neutrophil } \\
\text { predominance }\end{array}$ & $\begin{array}{l}\text { Neutrophil } \\
\text { predominance }\end{array}$ & Lymphocytes & $\begin{array}{l}28.9 \% \\
\text { neutrophils } \\
\text { and } 70.4 \% \\
\text { lymphocytes. }\end{array}$ & $\begin{array}{l}\text { Neutrophils } \\
87 \%\end{array}$ & $\begin{array}{l}\text { Neutrophils } \\
86 \%\end{array}$ & $\begin{array}{l}\text { Neut } \\
94 \%\end{array}$ \\
\hline & $\begin{array}{l}\text { WBC } \\
\text { count }\end{array}$ & $300 / \mathrm{mm} 3$ & 3140 & 3356 & $1600 / \mathrm{mm} 3$ & $5100 / \mathrm{mm} 3$ & 820 & $1300 / \mathrm{mm} 3$ & 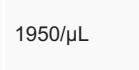 & $\begin{array}{l}2280 \times 109 \\
\text { cells/L, }\end{array}$ & 5965 \\
\hline \multirow{2}{*}{\multicolumn{2}{|c|}{$\begin{array}{l}\text { Brucella } \\
\text { agglutination test }\end{array}$}} & $1 / 2560$ & $\begin{array}{l}\text { Serum 1/160, } \\
\text { dialysate 1/60 }\end{array}$ & $\begin{array}{l}\text { Serum } \\
1 / 2560, \\
\text { dialysate } \\
\text { unknown }\end{array}$ & $\begin{array}{l}\text { Serum } 1 / 640 \text {, } \\
\text { dialysate } \\
\text { negative }\end{array}$ & $1 / 640$ & $1 / 5120$ & $1 / 1280$ & Not reported & Unknown & Nega \\
\hline & & & Rifampin p.o. & Doxycycline & & & $\begin{array}{l}\text { Rifampicin } \\
\text { p.o. }\end{array}$ & $\begin{array}{l}\text { Amikacin } \\
\text { intraperitoneal }\end{array}$ & $\begin{array}{l}\text { Levofloxacin } \\
\text { IP and } \\
\text { amikacin IP } \\
\text { for three } \\
\text { weeks then } \\
\text { minocycline }\end{array}$ & $\begin{array}{l}\text { Rifampicin } \\
\text { p.o., } \\
\text { doxycycline } \\
\text { p.o., } \\
\text { ciprofloxacin } \\
\text { p.o. }\end{array}$ & \\
\hline
\end{tabular}




\section{Cureus}

\begin{tabular}{|c|c|c|c|c|c|c|c|c|c|c|}
\hline Treatment & $\begin{array}{l}\text { Doxycycline } \\
\text { p.o. and } \\
\text { rifampicin p.o. } \\
\text { for six weeks }\end{array}$ & $\begin{array}{l}\text { and } \\
\text { doxycycline } \\
\text { p.o. for six } \\
\text { weeks }\end{array}$ & $\begin{array}{l}\text { p.o. and } \\
\text { rifampicin } \\
\text { p.o. for } 12 \\
\text { weeks }\end{array}$ & $\begin{array}{l}\text { Doxycycline } \\
\text { p.o. and } \\
\text { rifampicin p.o. } \\
\text { for six weeks }\end{array}$ & $\begin{array}{l}\text { Doxycycline } \\
\text { p.o. and } \\
\text { rifampicin p.o. } \\
\text { for six weeks }\end{array}$ & $\begin{array}{l}\text { ceftriaxone for } \\
45 \text { days, } \\
\text { doxycycline } \\
\text { was not } \\
\text { tolerated }\end{array}$ & $\begin{array}{l}\text { (IP), } \\
\text { doxycycline } \\
\text { p.o., and } \\
\text { rifampicin p.o. } \\
\text { for } 42 \text { days }\end{array}$ & $\begin{array}{l}\text { p.o., } \\
\text { rifampicin } \\
\text { p.o., and } \\
\text { levofloxacin } \\
\text { p.o. for a } \\
\text { total } \\
\text { treatment } \\
\text { duration of } \\
18 \text { weeks }\end{array}$ & $\begin{array}{l}\text { Rifampicin } \\
\text { was } \\
\text { discontinued } \\
\text { and } \\
\text { doxycycline } \\
\text { was replaced } \\
\text { by } \\
\text { minocycline } \\
\text { for } 12 \text { weeks }\end{array}$ & $\begin{array}{l}\text { Oral } \\
\text { rifam } \\
\text { and c } \\
\text { doxy }\end{array}$ \\
\hline Outcome & $\begin{array}{l}\text { PD catheter } \\
\text { removed. The } \\
\text { patient was } \\
\text { switched to } \\
\text { hemodialysis }\end{array}$ & $\begin{array}{l}\text { PD catheter } \\
\text { removed. The } \\
\text { patient was } \\
\text { switched to } \\
\text { hemodialysis }\end{array}$ & $\begin{array}{l}\text { PD catheter } \\
\text { removed. } \\
\text { The patient } \\
\text { was } \\
\text { switched to } \\
\text { hemodialysis }\end{array}$ & $\begin{array}{l}\text { PD was } \\
\text { resumed as } \\
\text { usual }\end{array}$ & $\begin{array}{l}\text { PD was } \\
\text { resumed as } \\
\text { usual }\end{array}$ & $\begin{array}{l}\text { PD was } \\
\text { resumed as } \\
\text { usual }\end{array}$ & $\begin{array}{l}\text { PD was } \\
\text { resumed as } \\
\text { usual }\end{array}$ & $\begin{array}{l}\text { PD was } \\
\text { resumed as } \\
\text { usual }\end{array}$ & $\begin{array}{l}\text { PD catheter } \\
\text { removed. The } \\
\text { patient was } \\
\text { switched to } \\
\text { hemodialysis }\end{array}$ & $\begin{array}{l}\text { PD n } \\
\text { resur } \\
\text { usua }\end{array}$ \\
\hline
\end{tabular}

TABLE 2: Reported cases of Brucella PD-related peritonitis.

PD: peritoneal dialysis; p.o.: per oral; IP: intraperitoneal.

Regarding clinical presentations, the most common presenting symptom was abdominal pain. Fever was present in four cases. Our patient presented both fever and abdominal pain. Most cases reported that there was a source for infection with Brucella, whereas in our case, the source of infection was not clear, with no obvious environmental risk factors. Her mother denied any history of ingestion of unpasteurized milk or contact with raw infected animal products, but she had received a blood transfusion a few months before presentation. The risk of transfusion-transmitted brucellosis has been reported [18]. Serology is the preferred method for diagnosing brucellosis when bacterial isolation is not possible, and serological testing is widely used in the diagnosis of brucellosis [19]. Turan Buzgan et al. reported that $1.1 \%$ of the patients remained seronegative [19]. As the peritoneal fluid showed a positive result for Brucella species after four days of growth, and the serum serology titer for Brucella revealed negative findings, we diagnosed the patient with seronegative Brucella peritonitis.

The PD catheter was removed in four patients with Brucella peritonitis [6,11,17]. However, our patient was successfully treated with antibiotics without the removal of the PD catheter. Niu et al. suggested that catheter removal should be considered for those patients with severe manifestations, who are unresponsive despite optimal treatment with intraperitoneal antibiotics and appropriate oral antibiotics [16]. Several studies have shown that it is necessary to use antibiotics for at least six weeks or more to avoid relapse or develop resistance [6]. The best results are observed when antimicrobial treatment is administered early in combination therapy with adequate dosing [16]. The treatment commonly used for children is age-specific. If the patient is aged greater than eight years, the treatment is complex, consisting of oral doxycycline and rifampicin. Children aged less than eight years are routinely treated with trimethoprim, sulfamethoxazole, and rifampicin [6,20]. In most of the reported cases, doxycycline and rifampicin were administered.

Levofloxacin or ciprofloxacin was added in some cases [16,17]. Ceftriaxone was administered to one patient, who could not tolerate doxycycline [14]. In our patient, oral rifampicin and doxycycline were highly effective.

\section{Conclusions}

In conclusion, Brucella peritonitis in a PD patient is rare but associated with excellent outcomes if treated appropriately. In PD-related peritonitis that does not respond to standard antibiotic treatment, Brucella peritonitis should be considered, particularly in endemic countries. The use of oral antibiotics is a good option for treatment unless the patient cannot tolerate it.

\section{Additional Information \\ Disclosures}

Human subjects: Consent was obtained or waived by all participants in this study. Conflicts of interest: In compliance with the ICMJE uniform disclosure form, all authors declare the following: Payment/services info: All authors have declared that no financial support was received from any organization for the submitted work. Financial relationships: All authors have declared that they have no financial relationships at present or within the previous three years with any organizations that might have an interest in the submitted work. Other relationships: All authors have declared that there are no other relationships or activities that could appear to have influenced the submitted work.

\section{Acknowledgements}

I would like to thank the pediatric nephrology team and pediatric infectious diseases team, as well as the research center at King Fahad Medical City, Riyadh, for their continuous support.

\section{References}

1. Cleper R, Davidovits M, Kovalski Y, Samsonov D, Amir J, Krause I: Peritonitis in a pediatric dialysis unit: local profile and implications. Isr Med Assoc J. 2010, 12:348-52. 
2. Liu FX, Gao X, Inglese G, Chuengsaman P, Pecoits-Filho R, Yu A: A global overview of the impact of peritoneal dialysis first or favored policies: an opinion. Perit Dial Int. 2015, 35:406-20. 10.3747/pdi.2013.00204

3. Klaus G: Prevention and treatment of peritoneal dialysis-associated peritonitis in pediatric patients . Perit Dial Int. 2005, 25:117-9. 10.1177/089686080502503830

4. Davenport A: Peritonitis remains the major clinical complication of peritoneal dialysis: the London, UK, peritonitis audit 2002-2003. Perit Dial Int. 2009, 29:297-302. 10.1177/089686080902900314

5. Corbel MI: Brucellosis in Humans and Animals. World Health Organization, Geneva, Switzerland; 2006.

6. Bakheet HG, Alnakhli HA: Brucellosis in Saudi Arabia: review of literature and epidemiology . J Trop Dis. 2019, 7:2-5.

7. Taskapan H, Oymak O, Sümerkan B, Tokgoz B, Utas C: Brucella peritonitis in a patient on continuous ambulatory peritoneal dialysis with acute brucellosis. Nephron. 2002, 91:156-8. 10.1159/000057618

8. Unal A, Sipahioglu MH, Kavuncuoglu F, Tokgoz B, Oymak O, Sumerkan B, Utas C: Peritoneal dialysis-related peritonitis caused by Brucella melitensis. Dial Transplant. 2009, 38:515-6. 10.1002/dat.20385

9. Colmenero JD, Reguera JM, Cabrera FP, Cisneros JM, Orjuela DL, Fernández-Crehuet J: Serology, clinical manifestations and treatment of brucellosis in different age groups. Infection. 1990, 18:152-6. 10.1007/BF01642103

10. Li PK, Szeto CC, Piraino B, et al.: ISPD peritonitis recommendations: 2016 update on prevention and treatment. Perit Dial Int. 2016, 36:481-508. 10.3747/pdi.2016.00078

11. Ozisik L, Akman B, Huddam B, Azap OK, Bilgic A, Sezer S, Ozdemir N: Isolated Brucella peritonitis in a CAPD patient. Am J Kidney Dis. 2006, 47:e65-6. 10.1053/j.ajkd.2006.01.028

12. Al-Qarhi R, Al-Dabbagh M: Brucella shunt infection complicated by peritonitis: case report and review of the literature. Infect Dis Rep. 2021, 13:367-76. 10.3390/idr13020035

13. Alothman A, Al Khurmi A, Al Sadoon S, AlHejaili F: Brucella peritonitis in a patient on peritoneal dialysis . Saudi J Kidney Dis Transpl. 2008, 19:428-30.

14. Solak Y, Biyik Z, Demircioglu S, Polat I, Genc N, Turkmen K, Turk S: Brucella peritonitis in peritoneal dialysis: a case report and review of the literature. Perit Dial Int. 2012, 32:126-30. 10.3747/pdi.2011.00056

15. Koz S, Sahin I, Kayabas U, Kuzucu C: Brucella and peritoneal dialysis related peritonitis: case report and review of literature. Clin Nephrol. 2014, 82:283-6. 10.5414/CN107828

16. Niu Q, Zhao H, Chen M, et al.: Brucella peritonitis in a patient on peritoneal dialysis: case report and literature review. Perit Dial Int. 2018, 38:S64-8. 10.3747/pdi.2018.00115

17. Bukhari M, Mohammed A, Al-Guraibi A, Shahata A, Al Ghamdi M: Lessons from the success and failures of peritoneal dialysis-related Brucella peritonitis in the last 16 years: case report and literature review. J Clin Nephrol. 2018, 2:057-61. 10.29328/journal.jcn.1001020

18. Wang $\mathrm{W}$, Liao $\mathrm{Q}, \mathrm{Wu} \mathrm{X}$, et al.: Potential risk of blood transfusion-transmitted brucellosis in an endemic area of China. Transfusion. 2015, 55:586-92. 10.1111/trf.12853

19. Buzgan T, Karahocagil MK, Irmak H, Baran AI, Karsen H, Evirgen O, Akdeniz H: Clinical manifestations and complications in 1028 cases of brucellosis: a retrospective evaluation and review of the literature. Int J Infect Dis. 2010, 14:e469-78. 10.1016/j.ijid.2009.06.031

20. Alshaalan MA, Alalola SA, Almuneef MA, Albanyan EA, Balkhy HH, AlShahrani DA, AlJohani S: Brucellosis in children: prevention, diagnosis and management guidelines for general pediatricians endorsed by the Saudi Pediatric Infectious Diseases Society (SPIDS). Int J Pediatr Adolesc Med. 2014, 1:40-6. 10.1016/j.ijpam.2014.09.004 